\title{
A novel genomic DNA library preparation method with low GC bias
}

\author{
S. Thomas Kelly ${ }^{1}$, Tsuneo Hakoyama ${ }^{2}$, Kie Kumaishi ${ }^{3}$, Haruka Okuda-Yabukami ${ }^{1}$, Sachi Kato ${ }^{1}$, \\ Makoto Hayashi², Aki Minoda ${ }^{*}$, and Yasunori Ichihashi ${ }^{3 *}$ \\ *Corresponding authors: akiko.minoda@riken.jp, yasunori.ichihashi@riken.jp \\ 1. RIKEN Center for Integrative Medical Sciences, Kanagawa 230-0045, Yokohama, Japan. \\ 2. RIKEN Center for Sustainable Resource Science, Yokohama, Kanagawa 230-0045, Japan \\ 3. RIKEN BioResource Research Center, Tsukuba, Ibaraki 305-0074, Japan
}

\begin{abstract}
The amount of input DNA available to prepare next-generation sequencing (NGS) libraries is often limited, which can lead to GC content bias and enrichment of specific genomic regions with currently available protocols. In this study, we used breath capture technology to incorporate sequencing adapters into DNA to develop a novel cost-effective protocol for the preparation of genomic DNA libraries. We performed a benchmarking experiment comparing our protocol with common commercially available kits for genomic DNA library preparation with input DNA amount in the range of 1 to $50 \mathrm{ng}$. Our protocol can generate high-quality genomic sequence data with a marked improvement in coverage breadth and low GC bias, in contrast to standard protocols. Further, our protocol reduces sample handling time and reagent costs, and requires comparatively fewer enzymatic steps relative to other protocols, making it suitable for a range of genomics applications.
\end{abstract}

\section{Introduction}

Next-generation sequencing (NGS) platforms have been used for a wide range of investigations in genomics and related applications. However, several library preparation methods require a high amount of input DNA, which may not be feasible for some applications (Li et al., 2019). For example, very low DNA yields are common in analyses such as chromatin immunoprecipitation (ChIP-seq; commonly performed for epigenomic analyses), degraded DNA from field samples (for example in forensic science, archaeology, and paleontology) (Gansauge and Meyer, 2013; Xavier and Parson, 2017), parasites and microorganisms that cannot be cultured (Nascimento et al., 2016), viruses (Malboeuf et al., 2013), very small insects (Cruaud et al., 2019), and metagenomics of environmental samples (Rinke et al., 2014). Low DNA input can also be an issue in single-cell analyses (Harada et al., 2019; Kaya-Okur et al., 2019), analyses using circulating fetal or tumor DNA (Mauger et al., 2020), and clinical studies with formalin-fixed paraffin-embedded tumor tissue samples (Munchel et al., 2015; Kader et al., 2016; Zhang et al., 2019).

Commercially available kits, such as TruSeq ${ }^{\circledR}$ Nano (Illumina), Nextera® (Illumina), and ThruPLEX® (Takara Bio) are popular for library preparation for NGS experiments with low sample input. TruSeq (Illumina) kits have been shown to produce high-quality data for RNA-Seq using low cDNA input compared to other protocols (Song et al., 2018; Sarantopoulou et al., 2019). TruSeq Nano remains one of the most popular and reliable library preparation techniques (Li et al., 2019; Pasquali et al., 2019) using a low sample input (as low as $1 \mathrm{ng}$ ) and higher sensitivity than TruSeq version 2 (Rhodes et al., 2014) or NEBNext $®$ Ultra. The Nextera genomic DNA library preparation kit that uses the $\mathrm{Tn} 5$ transpose can be used to prepare libraries rapidly using a low input at $1 \mathrm{ng}$ or lower with high sensitivity. However, the kit is costly and shows GC coverage and 
sequence biases compared to the TruSeq kits (Adey et al., 2010; Lan et al., 2015; Sato et al., 2019). Nextera kits are also susceptible to the presence of contamination or duplicates (Rinke et al., 2014), do not detect more variants than lower-cost alternatives (Pasquali et al., 2019), and the limited control of fragment size can introduce biases (Adey et al., 2010; Nascimento et al., 2016). The Rubicon Genomics ThruPLEX kit (Takara Bio) has been shown to have relatively high sensitivity for de novo genome assembly and reliably detects variants in low-input DNA (Chung et al., 2016; Nascimento et al., 2016; Mauger et al., 2020) and ChIP-Seq samples (Sundaram et al., 2016).

Because library preparation can be expensive, there have been several efforts to reduce the cost, including dilution of kits (Cruaud et al., 2019; Li et al., 2019) and utilization of cheaper alternatives (Dunham et al., 2019; Pasquali et al., 2019). Notably, it is possible to reduce the cost of preparation of genomic and transcriptomic libraries without compromising the variant and gene detection rates or data quality (Combs and Eisen, 2015; Pasquali et al., 2019). Numerous protocols have been developed to enable ChIP-Seq with a low input (Schmidl et al., 2015; KayaOkur et al., 2019; Handa et al., 2020). For example, ChIP-Seq studies in plant tissues would not be possible without these low-input techniques (Birkenbihl et al., 2017; Zheng and Gehring, 2019). As such, the community would benefit tremendously from a simple, fast, and low-cost alternative preparation technique.

The cost of library preparation can be reduced significantly using custom protocols compared to commercial kits (Kumar et al., 2012; Baym et al., 2015). Previously, we have demonstrated the utility of the Breath Adapter Directional sequencing (BrAD-seq) protocol for the preparation of RNA-Seq libraries (Townsley et al., 2015; Ichihashi et al., 2018). The BrAD-Seq technique is a streamlined, ultra-simple, and fast library preparation method that uses breath-capture technology to incorporate strand-specific adapters, enabling large-scale transcriptomic studies at a lower cost. In this study, we adapted the breath capture technology in the BrAD-Seq protocol (shown in Fig. 1) to prepare low sample input libraries for DNA sequencing and compared the results obtained with commercial kits, namely Illumina TruSeq and Rubicon ThruPLEX. The revised protocol has the advantages of lower cost in consumables (Table 1) and a shorter protocol. A cheaper genome sequencing alternative for low input and poor-quality samples would expand the range of feasible applications in genomics and epigenomics experiments and increase sample sizes (Lakens, 2021).

Protocols that reduce GC bias in libraries are necessary since variations in the GC content between genomes can cause biases in the analyses, requiring greater sequencing depth for de novo genome assembly (Kozarewa et al., 2009; Benjamini and Speed, 2012; Chen et al., 2013). GC bias can also be problematic for variant calling, metagenomics, and quantitative analyses by ChIP-Seq, as regions with low GC content will be under-represented in low-coverage libraries (Benjamini and Speed, 2012; Rinke et al., 2016; Sato et al., 2019; Browne et al., 2020). Avoiding GC bias is also important when working with ancient DNAs, as it reduces the contamination of GC-rich microbial DNA (Dabney and Meyer, 2012). Amplification-free techniques have been developed previously to reduce GC bias; however, they require high amounts of input material, making GC bias a widespread issue in published data with low input amounts (Chen et al., 2013).

Here we demonstrate the application of the BrAD-Seq method to genomic DNA by comparing it to commercially available kits by criteria such as coverage breadth and GC bias. 


\section{Methods}

\section{Sample materials and preparation}

Genomic DNA samples were obtained from plants (A. thaliana) and fruit flies (D. melanogaster). Wild-type thale cress Col-0 genomic DNA (BioChain Institute Inc. BCH \#D1634310-5) was purchased from Cosmo Bio (Tokyo, Japan). Genomic DNA was extracted from D. melanogaster ( $w^{1118}$ mutant strain BDGC \#5905).

\section{DNA fragmentation}

The plant genomic DNA samples were diluted with TE (10 mM Tris-HCl pH 8.0, 1 mM EDTA) to $10 \mathrm{ng} / \mu \mathrm{l}$, and $20 \mu \mathrm{l}$ of the diluted DNA samples were sheared by heat treatment $\left(95^{\circ} \mathrm{C}, 45 \mathrm{~min}\right)$ into fragments ranging from $500-1000 \mathrm{bp}$. The fragmented DNA was purified by the addition of $30 \mu \mathrm{l}$ Agencourt AMPure XP beads (Beckman Coulter) and mixed by pipetting. After $5 \mathrm{~min}$, the samples were placed on a magnetic tray and the supernatants were removed. Pellets were washed twice with $80 \%$ ethanol. Residual ethanol was removed completely, and the pellets were air-dried for $2 \mathrm{~min}$. The fragmented DNA samples were eluted with $10 \mu \mathrm{l}$ of $10 \mathrm{mM}$ Tris- $\mathrm{HCl}(\mathrm{pH}$ 7.5) and quantified using a Nanodrop (Thermo Scientific). Samples were aliquoted into $1 \mathrm{ng}, 10$ ng, and 50 ng amounts. Samples were amplified by PCR with the cycling details described in Table S1 after preparation with each library protocol.

Fruit fly genomic DNA samples were prepared with the Focused-ultrasonicator S220 (Covaris; duty factor $10 \%$, peak incident power(w) 140, cycle/burst 200, time $80 \mathrm{~s}$ ) into fragments ranging from 300-400 bp. The samples were aliquoted into $1 \mathrm{ng}$ and $10 \mathrm{ng}$ aliquots and were amplified by PCR with the cycling details described in Table S2 after preparation with each library protocol.

\section{Generation of BrAD-Seq libraries for genomic DNA}

The full BrAD-Seq protocol for genome DNA was deposited at protocols.io (dx.doi.org/10.17504/protocols.io.bsx9nfr6). The adapter and primer sequences used are listed in Table S3.

3' adapter priming

The fragmented DNA samples were aliquoted into different amounts $(1 \mathrm{ng}, 10 \mathrm{ng}$, and $50 \mathrm{ng}$ for plants and $1 \mathrm{ng}$ and $10 \mathrm{ng}$ for flies) to evaluate the effect of DNA amount on the sequencing library quality. The DNA fragments were used as templates to produce libraries that had adapter sequences for Illumina sequencing at the $5^{\prime}$ and $3^{\prime}$ ends. After denaturing the double-stranded DNA (dsDNA) to single-stranded DNA (ssDNA) by heat treatment, the PE1 adapter sequence was added to the 3' ends of the ssDNA using DNA polymerase in a $15 \mu \mathrm{l}$ reaction mixture containing $1.5 \mu \mathrm{l} 10 \times$ Ex Taq buffer, $1.2 \mu \mathrm{l}$ dNTPs ( $2.5 \mathrm{mM}$ each), $0.075 \mu \mathrm{l}$ (0.375 units) of Ex Taq (Takara), $5 \mu \mathrm{l}$ of the fragmented DNA, $1 \mu \mathrm{l}$ of $5 \mu \mathrm{M}$ 3' adapter oligo (L-3ILL-N8.2 in Table S3), and $6.225 \mu \mathrm{l}$ $\mathrm{H}_{2} \mathrm{O}$. The mixtures were incubated in a thermal cycler with the following program: $94^{\circ} \mathrm{C}$ for $2 \mathrm{~min}$, $45^{\circ} \mathrm{C}$ for $10 \mathrm{~min}, 42^{\circ} \mathrm{C}$ for $10 \mathrm{~min}, 72^{\circ} \mathrm{C}$ for $5 \mathrm{~min}$, and hold at $4^{\circ} \mathrm{C}$. The synthesized DNA was purified by addition of $5 \mu \mathrm{l} 50 \mathrm{mM}$ EDTA (pH 8.0) and $30 \mu \mathrm{l}$ Agencourt AMPure XP beads to each sample and mixed by pipetting. After $5 \mathrm{~min}$, the sample was placed on a magnetic tray and the supernatant was removed. The pellets were washed twice with $80 \%$ ethanol. The residual ethanol was completely removed and air-dried for $2 \mathrm{~min}$. 
Introduction of the adapter sequence into the $5^{\prime}$ ends of ssDNA fragments was performed using breath capture technology with a 5' double-stranded adapter oligo (Townsley et al., 2015; Ichihashi et al., 2018). The 5' double-stranded adapter oligo was prepared by hybridization using $10 \mu \mathrm{l}$ each of $10 \mathrm{mM}$ oligos 5pSense8n and 5pAnti (described in Table S3) in $80 \mu \mathrm{l}$ of $\mathrm{H}_{2} \mathrm{O}$. The mixture was incubated in a thermal cycler with the following program: $94^{\circ} \mathrm{C}$ for $1 \mathrm{~min} ;\left(94^{\circ} \mathrm{C}\right.$ for $10 \mathrm{~s}) \times 60$ with $-1^{\circ} \mathrm{C} /$ cycle, $20^{\circ} \mathrm{C}$ for $1 \mathrm{~min}$, and hold at $4^{\circ} \mathrm{C}$. The bead-bound DNA samples were eluted with $4 \mu \mathrm{l}$ of $10 \mathrm{mM} \mathrm{5}$ ' double-stranded adapter oligo. Subsequently, $6 \mu \mathrm{l}$ of the following mixtures were added: $1 \mu \mathrm{l} 10 \times$ Pol I buffer, $0.25 \mu \mathrm{l}$ dNTPs ( $25 \mathrm{mM}$ each, Thermo Scientific), 0.25 $\mu \mathrm{l}$ DNA polymerase I (Thermo Scientific) and $4.5 \mu \mathrm{l} \mathrm{H}_{2} \mathrm{O}$. The mixture was incubated at $25^{\circ} \mathrm{C}$ for $15 \mathrm{~min}$. The samples were size-selected by the addition of $10 \mu \mathrm{l}$ of $50 \mathrm{mM}$ EDTA and $30 \mu \mathrm{l}$ of ABR buffer (15\% PEG 8000, $2.5 \mathrm{M} \mathrm{NaCl}$ ) and mixed by pipetting. The samples were allowed to stand for $5 \mathrm{~min}$ and were subsequently placed on a magnetic tray. The supernatant was removed, and the pellets were washed twice with $80 \%$ ethanol. The pellets were air-dried for $2 \mathrm{~min}$ and resuspended in $30 \mu \mathrm{l}$ of $10 \mathrm{mM}$ Tris- $\mathrm{HCl}$ ( $\mathrm{pH} 7.5$ ).

Enrichment and index sequence addition

Full-length adapter sequences were added to the libraries and amplified by PCR using the primers PE1 and PE2 (Table S3). The enrichment and adapter extension steps were performed in a reaction mixture containing $2 \mu \mathrm{l} 10 \times$ Ex Taq buffer, $1.6 \mu \mathrm{l}$ dNTPs $(2.5 \mathrm{mM}$ each), $0.1 \mu \mathrm{l}$ Ex Taq (Takara, cat\# RR001A), $4.3 \mu \mathrm{l} \mathrm{H} \mathrm{H}_{2}, 10 \mu \mathrm{l}$ breath captured DNA, $1 \mu \mathrm{l}$ of $2 \mu \mathrm{M}$ PE1 primer, and $1 \mu \mathrm{l}$ of $2 \mu \mathrm{M}$ PE2 primer. The reaction mixtures were incubated in a thermal cycler with the following program: $94^{\circ} \mathrm{C}$ for $2 \mathrm{~min} ; 94^{\circ} \mathrm{C}$ for $30 \mathrm{~s} ; 65^{\circ} \mathrm{C}$ for $30 \mathrm{~s} ; 72^{\circ} \mathrm{C}$ for $30 \mathrm{~s}$ ) $\times 16-21$ (see Tables S1 and S2); $72^{\circ} \mathrm{C}$ for $7 \mathrm{~min}$; and hold at $4{ }^{\circ} \mathrm{C}$. The PCR products were cleaned with $16 \mu$ Agencourt AMPure XP beads and the samples were mixed by pipetting. After $5 \mathrm{~min}$, the samples were placed on a magnetic tray and the supernatants were removed. The pellets were washed twice with $80 \%$ ethanol. Residual ethanol was removed completely using a $10 \mu$ pipette tip, and the sample was air-dried for $2 \mathrm{~min}$. The libraries were eluted using $10 \mu \mathrm{l} \mathrm{10mM} \mathrm{Tris-HCl} \mathrm{(pH7.5).}$

\section{Generation of Illumina libraries using commercially available library preparation kits} TruSeq® Nano DNA LT Library Preparation Kit (Illumina) for plant samples and TruSeq® ChIP Sample Preparation Kit (Illumina) for fly samples were used according to the manufacturer's protocols. PCR cycling was performed as described in Tables S1 and S2. For the Rubicon Genomics ThruPLEX® DNA-seq Kit: High Performance Library Preparation for Illumina® NGS Platforms (currently available from Takara Bio USA), the manufacturer's protocol was followed.

\section{Sequencing on the Illumina platform}

The plant samples were sequenced on the Illumina MiSeq (single-end sequencing with 68 cycles and $8 \mathrm{bp}$ index) with at least 1 million reads for each sample obtained.

The fruit fly samples were sequenced on an Illumina NovaSeq 6000 (paired-ends with 151 cycles and 8 bp index). Due to sample dilution factors, some samples were sequenced to a significantly greater depth than others.

\section{Bioinformatics analysis}

A reproducible bioinformatics pipeline was developed in-house to ensure that all samples were processed using the same parameters. These were all run on the same architecture using a Debian 9 Linux server with an SGE job scheduler. 
Samples were demultiplexed using bcl2fastq 2.17.1.14 (Illumina, Inc., 2017). Quality checks were performed on the raw data using FastQC v0.11.8 (Andrews, 2010) and MultiQC v1.7 (Ewels et al., 2016) using Python 3.6.8 (van Rossum and Drake, 2009). Due to differences in the number of reads for each library, raw FASTQ files were downsampled using seqtk $1.2(\mathrm{Li}, 2016)$ to the same depth of 1 million reads. Analysis of the fruit fly samples was performed with 1,000,000 reads for direct comparison with the plant samples.

Reads were trimmed using the FASTX-Toolkit 0.0.14 (Hannon, 2010) to remove the first eight bases as described in Townsley et al. (2015). All samples were trimmed to the same length for comparison purposes. Reads were filtered by quality using Trimmomatic 0.39 (Bolger et al., 2014) running on Java openjdk 1.8.0, because the algorithm supports processing of single and paired-end reads. All samples were filtered using the same quality threshold.

Samples were aligned to the respective reference genomes using Bowtie2 2.3.0 (Langmead and Salzberg, 2012) using default parameters. The TAIR10 reference for Arabidopsis thaliana was obtained from the Arabidopsis Information Resource database (Lamesch et al., 2011). The Drosophila melanogaster reference Berkeley Drosophila Genome Project assembly BDGP6.22 (dos Santos et al., 2015) was obtained from Ensembl release 96 (Cunningham et al., 2019).

Coverage and summary statistics were computed using samtools 1.3.1 (Li et al., 2009), bedtools 2.26.0 (Quinlan and Hall, 2010), and custom shell scripts. Data were processed and plotted using R 3.6.2 (R Core Team, 2019). The data.table 1.12.8 (Dowle and Srinivasan, 2019) and vroom 1.2.0 (Hester and Wickham, 2020) R packages were instrumental for processing large datasets efficiently. Library complexity was computed using preseq 3.0.2 (Daley and Smith, 2013). GC bias was computed using DeepTools v3.5.0 (Benjamini and Speed, 2012; Ramírez et al., 2016)

Non-overlapping sliding (stepping) windows of 10,000 nucleotides and base frequencies at nucleotide resolution were computed using bedtools 2.26.0 (Quinlan and Hall, 2010). The coverage depth at the nucleotide resolution was computed using samtools 1.3 .1 (Li et al., 2009) and sliding windows were computed in R 3.6.2 (R Core Team, 2019). Coverage depth was normalized by window length to account for truncated windows at the ends of chromosomes and scaffolds. The GC content was computed from base frequencies, and summary statistics for coverage depth were computed using GNU awk 4.1.4 (Aho et al., 1988) and averaged in sliding windows in R 3.6.2 (R Core Team, 2019). Least-squares linear regression analysis was performed $\left(y \sim \beta_{1} x+\beta_{0}\right)$, where $y$ is the adjusted coverage depth and $x$ is the GC content for each $10,000 \mathrm{bp}$ sliding window for the genome. $\beta_{1}$ is the coefficient for the magnitude of the linear relationship between the GC content and coverage depth.

\section{Results}

228 By modifying the breath capture technology used in the BrAD-Seq protocol that was originally 229 developed for RNA-seq, we succeeded in adapting the protocol for DNA sequencing (Fig. 1). To assess the utility of the novel protocol for genomic DNA sequencing, we compared its performance to the commercially available kits TruSeq (Illumina) and ThruPLEX (Rubicon Genomics) using plant Arabidopsis thaliana (A. thaliana) DNA and fruit fly Drosophila melanogaster (D. melanogaster) DNA. These kits have been shown to perform well with low DNA input (Sato et al., 2019; Mauger et al., 2020) and are popular for WGS or ChIP-Seq analysis. 


\section{BrAD-Seq generates libraries with high coverage breadth with less organelle genomes} Plant and fruit fly genomic DNA was processed using the BrAD-Seq protocol in duplicate and sequenced on the Illumina MiSeq and NovaSeq platforms, respectively. The raw reads were down-sampled to 1 million reads per sample to carry out comparisons (Fig. 2) with respect to the mapping rate of filtered reads (Fig. 2a and Fig. 2b), coverage fold-depth (mean read depth) (Fig. $2 \mathrm{c}$ and Fig. 2d), coverage breadth (proportion of the genome with three or more reads) (Fig. 2e and Fig. 2f), and uniquely mapped reads (Fig. $2 \mathrm{~g}$ and $2 \mathrm{~h}$ ). Of note, due to poorer quality in Read $2 \mathrm{~s}$, both the mapping and unique mapping rates were lower with paired-end sequencing data (from flies) compared with single-end read sequencing data (plant, Fig. S1). Thus, fly data from Read 1 were analyzed further for direct comparison with the plant data. The proportion of mapped reads were similar between the raw data and the down-sampled sets (Tables S4 and S5), indicating the results are representative.

Our analysis shows BrAD-Seq libraries exhibit higher coverage breadth than the libraries prepared by other kits (Fig. 2e and Fig. 2f), indicating that BrAD-Seq libraries contain more sequences representing a wider range of genomic loci. This was more evident with the plant genomic DNA than with the fly genome ( $\sim 3$-fold higher for the plant and $\sim 1$.2-fold higher for the fly). In addition, BrAD-Seq libraries showed a higher unique mapping rate with the plant genomic DNA (Fig. 2g, h, and Table S4, S5). Therefore, BrAD-Seq appears to capture and cover more regions of the genome than other techniques, the beneficial effects of which appear to be more enhanced with the plant DNA. This was confirmed by determining proportions of mapped reads for each chromosome where we found that BrAD-Seq more accurately captured the expected ratio of mapped reads on $A$. thaliana chromosome 1 (87.1\% of what is expected for chromosome length) compared with other techniques (34.9\% in TruSeq; $31.7 \%$ in ThruPLEX, Fig. 3a). In addition, BrAD-Seq was less biased for multi-copy plastid genomes (Tables S6 and S7), exhibiting fewer mapped reads in plant chloroplasts compared with TruSeq $(t=6.97, p=0.002$ ) or ThruPLEX $\left(\mathrm{t}=47.78, \mathrm{p}=4.38 \times 10^{-4}\right)$ and fly mitochondria compared with TruSeq $(\mathrm{t}=73.17, \mathrm{p}=$ $\left.1.36 \times 10^{-12}\right)$ or ThruPLEX $\left(\mathrm{t}=32.18, \mathrm{p}=9.48 \times 10^{-10}\right)$.

\section{BrAD-Seq shows tolerance for variability in GC content}

We next examined library complexity by resampling raw data and computing the number of unique molecules obtained (using preseq). Whereas the input amount of plant genomic DNA had a relatively small impact on library complexity, clear differences were observed between the protocols (Fig. 4a). The BrAD-Seq samples had a higher library complexity, which may be explained by the higher coverage breadth (Fig. 2e) and unique mapping rates (Fig. 2g). However, this was not replicated with the fly genomic DNA, which unexpectedly showed low library complexity (Fig. 4b).

In respect to the GC content, the plant libraries had a higher GC content than the genome average (36\%) with TruSeq libraries showing higher GC content and variance than others (Table S8). The fly libraries had a similar mean (42\%) and standard deviation to the genome (Table S9). GC bias was computed from aligned reads using DeepTools v3.5.0 for a fragment size of $300 \mathrm{bp}$ (Benjamini and Speed, 2012; Ramírez et al., 2016). Compared to the statistically expected distribution, BrAD-Seq libraries exhibited less bias towards high GC regions of the plant genome (Fig. 4c), but all techniques showed less bias in the fly samples (Fig. 4d). 
Regions with high GC content can be biased towards higher coverage depths due to lower annealing temperatures. Therefore, a linear relationship is expected between coverage and GC content for GC-biased techniques (Chen et al., 2013). Such bias was more pronounced in TruSeq and ThruPLEX libraries than in BrAD-Seq, and BrAD-Seq libraries did not show a linear trend or elliptical distribution indicative of GC bias (Fig. 5). This result is supported by the lower effect size of the GC content with BrAD-Seq compared to others measured by the slope $\left(\beta_{1}\right)$ of the leastsquares linear regression analysis (Table 2). Taken together, these results show that the BrADSeq protocol has less bias for GC content compared to the currently available low-input Illumina TruSeq and Rubicon ThruPLEX library preparation kits.

\section{Discussion}

Here, we present a fast, simple, and low-cost novel library preparation protocol for genomic DNA that can be used with low input amounts. The BrAD-Seq protocol is compatible with the Illumina sequencing platform and can be used for a wide range of species and applications. The BrAD-Seq protocol provides higher quality libraries, which are less biased towards multi-copy plastids and GC-rich regions of the genome than commercially available kits specific for low DNA inputs. The novel method appears to be especially advantageous for sequencing analysis for plants (e.g., $A$. thaliana), based on observations of higher unique mapping rates, less enrichment for plastid genes, and low bias for fragments with high GC content. It should also be readily applied to epigenomic analyses such as ChIP-Seq, chromosome conformation capture, and DNA footprinting, which tend to yield low amounts of DNA. Because sequence quality was higher for single-end sequencing than paired-end reads in the BrAD-Seq libraries due to the lower quality of read 2, single-end sequencing is recommended although paired-end sequencing can still be performed. It is of note that BrAD-Seq libraries appear to have lower read quality and mapping rates; however, sequencing at a sufficient depth can overcome this drawback and is arguably offset by reduced coverage bias, fast turn-around time, and reduced consumables costs.

To summarize, we present the BrAD-Seq protocol which has a higher coverage breadth, library complexity, and low GC bias as demonstrated in plant samples. Therefore, BrAD-Seq addresses the need for a low-cost, low-input technique with low GC bias in plants and other species with low GC content in the genome. By reducing costs (Lakens et al., 2021), the novel method improves the possibility of carrying out genomics studies with a larger number of samples in a diverse range of species.

\section{Data Availability}

All sequencing data were deposited in the NCBI sequence read archive (SRA) BioProject

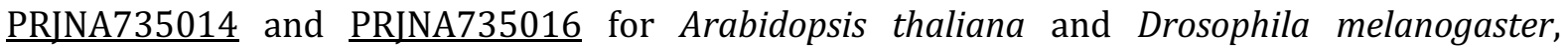
respectively.

\section{Conflicts of interest}

The authors declare no conflicts of interest exist.

\section{Acknowledgments} (DGRC \#5905). Sequencing was performed at the RIKEN IMS internal facilities and was outsourced to Takara Bio Japan K.K. We thank Dr. Shungo Kobori for his advice on the data analysis. This work was supported by a JSPS KAKENHI Grant-in-Aid for Scientific Research on 
329 Innovative Areas "Principles of pluripotent stem cells underlying plant vitality" (JP17H06470) to

330 AM, the Cabinet Office, Government of Japan, Cross-ministerial Moonshot Agriculture, Forestry

331 and Fisheries Research and Development Program, "Technologies for Smart Bio-industry and 332 Agriculture" (funding agency: Bio-oriented Technology Research Advancement Institution) to YI.

\section{Author's contributions}

$335 \mathrm{TH}, \mathrm{KH}$, and YI developed and optimized the novel experimental technique to prepare BrAD-Seq 336 libraries. HO-Y and SK prepared the libraries using the commercial kits. STK, AM, and YI designed 337 the experiments and prepared the manuscript. STK performed all data processing and 338 bioinformatic analyses. AM and YI oversaw the project. 


\section{References}

Adey A, Morrison HG, Asan, Xun X, Kitzman JO, Turner EH, Stackhouse B, MacKenzie AP, Caruccio NC, Zhang X, Shendure J (2010) Rapid, low-input, low-bias construction of shotgun fragment libraries by high-density in vitro transposition. Genome Biology 11(12): R119

Aho AV, Kernighan BW, Weinberger PJ (1988) The AWK programming language. Addison-Wesley Publishing Company. ISBN 9780201079814

Andrews S (2010) FastQC: A quality control tool for high-throughput sequence data. Available online at: http://www.bioinformatics.babraham.ac.uk/projects/fastqc

Baym M, Kryazhimskiy S, Lieberman TD, Chung H, Desai MM, Kishony R (2015) Inexpensive multiplexed library preparation for megabase-sized genomes. PLOS ONE 10(5): e0128036. https://doi.org/10.1371/journal.pone.0128036

Benjamini Y, Speed TP (2012) Summarizing and correcting the GC content bias in highthroughput sequencing. Nucleic Acids Research 40(10): e72 https://doi.org/10.1093/nar/gks001

Birkenbihl RP, Kracher B, Roccaro M, Somssich IE (2017) Induced genome-wide binding of three

Arabidopsis WRKY transcription factors during early MAMP-triggered immunity. Plant Cell 29(1): 20-38

Bolger AM, Lohse M, Usadel B (2014) Trimmomatic: A flexible trimmer for Illumina sequence data. 364 Bioinformatics 30(15): 2114-2120. https://doi.org/10.1093/bioinformatics/btu170

Browne PD, Nielsen TK, Kot W, Aggerholm A, Gilbert MTP, Puetz L, Rasmussen M, Zervas A, Hansen LH (2020) GC bias affects genomic and metagenomic reconstructions, underrepresenting GC-poor organisms. GigaScience 9(2): giaa008 https://doi.org/10.1093/gigascience/giaa008

Chen YC, Liu T, Yu CH, Chiang TY, Hwang CC (2013) Effects of GC Bias in next-generationsequencing data on de novo genome assembly. PLOS ONE 8(4): e62856. https://doi.org/10.1371/journal.pone.0062856

Chung J, Son DS, Jeon HJ, Kim KM, Park G, Ryu GH, Park WY, Park D (2016) The minimal amount of starting DNA for Agilent's hybrid capture-based targeted massively parallel sequencing. Scientific Reports 6: 26732 https://doi.org/10.1038/srep26732

Combs PA, Eisen MB (2015) Low-cost, low-input RNA-seq protocols perform nearly as well as high-input protocols. PeerJ 3: e869 DOI 10.7717/peerj.869 enrichment in chalcid wasps used for biocontrol. Molecular Ecology Resources 19(3): 702-710 
Cunningham F, Achuthan P, Akanni W, Allen J, Amode MR, Armean IM, Bennett R, Bhai J, Billis K, Boddu S et al. (2019) Ensembl 2019. Nucleic Acids Research 47(D1): D745-D751. https://doi.org/10.1093/nar/gky1113

Dabney J, Meyer M (2012) Length and GC-biases during sequencing library amplification: A comparison of various polymerase-buffer systems with ancient and modern DNA sequencing libraries. BioTechniques 52(2): 87-94 https://doi.org/10.2144/000113809

Dahl JA, Gilfillan GD (2018) How low can you go? Pushing the limits of low-input ChIP-seq. Briefings in Functional Genomics 17(2): 89-95, https://doi.org/10.1093/bfgp/elx037

Daley T, Smith AD (2013) Predicting the molecular complexity of sequencing libraries. Nature

dos Santos G, Schroeder AJ, Goodman JL, Strelets VB, Crosby MA, Thurmond J, Emmert DB, Gelbart WM, FlyBase Consortium (2015) FlyBase: Introduction of the Drosophila melanogaster Release 6 reference genome assembly and large-scale migration of genome annotations. Nucleic Acids Research Release 6 43(Database issue): D690-D697. https://doi.org/10.1093/nar/gku1099

Dowle M, Srinivasan A (2019) data.table: Extension of 'data.frame'. R package version 1.12.8. Available online at: https://CRAN.R-project.org/package=data.table

Dunham J, Angermayer C, Harkins T (2019) A 12 minute, single tube, nanogram input. Library prep. Journal of Biomolecular Techniques 30(Suppl): S1

Ewels P, Magnusson M, Lundin S, Käller M (2016) MultiQC: Summarize analysis results for multiple tools and samples in a single report. Bioinformatics 32(19): 3047-3048. https://doi.org/10.1093/bioinformatics/btw354

413

414

Gansauge MT, Meyer M (2013) Single-stranded DNA library preparation for the sequencing of ancient or damaged DNA. Nature Protocols 8(4): 737-748:/doi.org/10.1038/nprot.2013.038

Hannon GJ (2010) FASTX-Toolkit: FASTQ/A short-reads pre-processing tools. Available online at: http://hannonlab.cshl.edu/fastx toolkit

Harada A, Maehara K, Handa T, Arimura Y, Nogami J, Hayashi-Takanaka Y, Shirahige K, Kurumizaka H, Kimura H, Ohkawa Y (2019) A chromatin integration labelling method enables epigenomic profiling with lower input. Nature Cell Biology 21(2): 287-296. https://doi.org/10.1038/s41556-018-0248-3

Hester J, Wickham H (2020) vroom: Read and Write Rectangular Text Data Quickly R package 1.2.0. Available online at: https://CRAN.R-project.org/package=vroom 
Ichihashi Y, Fukushima A, Shibata A, Shirasu K (2018) High impact gene discovery: Simple-Strand Specific mRNA Library construction and differential regulatory analysis based on gene coexpression network. Methods in Molecular Biology 1830: 163-189

Illumina, Inc. (2017) bcl2fastq BCL to FASTQ file converter. version 2.17.1.14. Available online at: https://supportillumina.com/sequencing/sequencing software/bcl2fastq-conversionsoftware.html

Kader T, Goode DL, Wong SQ, Connaughton J, Rowley SM, Devereux L, Byrne D, Fox SB, Mir Arnau GM, Tothill RW, Campbell IG, Gorringe KL (2016) Copy number analysis by low coverage whole genome sequencing using ultra low-input DNA from formalin-fixed paraffin embedded tumor tissue. Genome Medicine 8(1): 121. https://doi.org/10.1186/s13073-016-0375-z

Kaya-Okur HS, Wu SJ, Codomo CA, Pledger ES, Bryson TD, Henikoff JG, Ahmad K, Henikoff S (2019) CUT\&Tag for efficient epigenomic profiling of small samples and single cells. Nature Communications 10(1): 1930 https://doi.org/10.1038/s41467-019-09982-5

Kozarewa I, Ning Z, Quail MA, Sanders MJ, Berriman M, Turner DJ (2009) Amplification-free Illumina sequencing-library preparation facilitates improved mapping and assembly of $(\mathrm{G}+\mathrm{C})$ biased genomes. Nature Methods 6(4): 291-295. https://doi.org/10.1038/nmeth.1311

Kumar R, Ichihashi Y, Kimura S, Chitwood DH, Headland LR, Peng J, Maloof JN, Sinha NR (2012) A high-throughput method for Illumina RNA-seq library preparation. Frontiers in Plant Science 3: 202 https://doi.org/10.3389/fpls.2012.00202

Lakens D (2021) Sample size justification. PsyArXiv doi: https://doi.org/10.31234/osf.io/9d3yf, 4 January 2021, preprint not peer-reviewed

Lamesch P, Berardini TZ, Li D, Swarbreck D, Wilks C, Sasidharan R, Muller R, Dreher K, Alexander DL, Garcia-Hernandez M, Karthikeyan AS, Lee CH, Nelson WD, Ploetz L, Singh S, Wensel A, Huala E (2012) The Arabidopsis Information Resource (TAIR): Improved gene annotation and new tools. Nucleic Acids Research 40(Database issue): D1202-D1210. https://doi.org/10.1093/nar/gkr1090

Lan JH, Yin Y, Reed EF, Moua K, Thomas K, Zhang Q (2015) Impact of three Illumina library construction methods on GC bias and HLA genotype calling. Human Immunology 76(2-3): 166175. https://doi.org/10.1016/i.humimm.2014.12.016

Langmead B, Salzberg SL (2012) Fast gapped-read alignment with Bowtie 2. Nature Methods 9(4): 357-359. https://doi.org/10.1038/nmeth.1923

Li H (2016) Seqtk: Toolkit for processing sequences in FASTA/Q formats. version 1.2. Available online at: https://github.com/lh3/seqtk

Li H, Handsaker B, Wysoker A, Fennell T, Ruan J, Homer N, Marth G, Abecasis G, Durbin R, 1000 Genome Project Data Processing Subgroup (2009) The Sequence Alignment/Map format and SAMtools. Bioinformatics 25(16): 2078-2079. https://doi.org/10.1093/bioinformatics/btp352 
Malboeuf CM, Yang X, Charlebois P, Qu J, Berlin AM, Casali M, Pesko KN, Boutwell CL, DeVincenzo

JP, Ebel GD, Allen TM, Zody MC, Henn MR, Levin JZ (2013) Complete viral RNA genome sequencing of ultra-low copy samples by sequence-independent amplification. Nucleic Acids Research 41(1): e13. doi: https://doi.org/10.1093/nar/gks794

Mauger F, Horgues C, Pierre-Jean M, Oussada N, Mesrob L, Deleuze JF (2020) Comparison of commercially available whole-genome sequencing kits for variant detection in circulating cellfree DNA. Scientific Reports 10(1): 6190. https://doi.org/10.1038/s41598-020-63102-8

Mora-Castilla S, To C, Vaezeslami S, Morey R, Srinivasan S, Dumdie JN, Cook-Andersen H, Jenkins J, Laurent LC (2016) Miniaturization technologies for efficient single-cell library preparation for next-generation sequencing. Journal of Laboratory Automation 21(4): 557-567. doi: https://doi.org/10.1177/2211068216630741

Munchel S, Hoang Y, Zhao Y, Cottrell J, Klotzle B, Godwin AK, Koestler D, Beyerlein P, Fan JB, Bibikova M, Chien J (2015) Targeted or whole genome sequencing of formalin fixed tissue samples: Potential applications in cancer genomics. Oncotarget 6(28): 25943-25961. https://doi.org/10.18632/oncotarget.4671

496

497

498

499

500

501

502

503

504

505

506

507

508

509

510

511

512

513

514

515

516

517

518

519

520

521

522

523

Nascimento FS, Wei-Pridgeon Y, Arrowood MJ, Moss D, da Silva AJ, Talundzic E, Qvarnstrom Y (2016) Evaluation of library preparation methods for Illumina next generation sequencing of small amounts of DNA from foodborne parasites. Journal of Microbiological Methods 130: 23-26 https://doi.org/10.1016/i.mimet.2016.08.020

Pasquali F, Do Valle I, Palma F, Remondini D, Manfreda G, Castellani G, Hendriksen RS, De Cesare A (2019) Application of different DNA extraction procedures, library preparation protocols and sequencing platforms: Impact on sequencing results. Heliyon 5(10): e02745 https://doi.org/10.1016/j.heliyon.2019.e02745

Quinlan AR, Hall IM (2010) BEDTools: A flexible suite of utilities for comparing genomic features. Bioinformatics 26(6): 841-842. https://doi.org/10.1093/bioinformatics/btq033

R Core Team (2019) R: A language and environment for statistical computing. Available online at: https://www.R-project.org/. R Foundation for Statistical Computing, Vienna, Austria

Ramírez F, Ryan DP, Grüning B, Bhardwaj V, Kilpert F, Richter AS, Heyne S, Dündar F, Manke T (2016) deepTools2: A next generation web server for deep-sequencing data analysis. Nucleic Acids Research 44(W1): W160-W165 https://doi.org/10.1093/nar/gkw257

Rhodes J, Beale MA, Fisher MC (2014) Illuminating choices for library prep: A comparison of library preparation methods for whole genome sequencing of Cryptococcus neoformans using Illumina HiSeq. PLOS ONE 9(11): e113501. https://doi.org/10.1371/journal.pone.0113501

Rinke C, Low S, Woodcroft BJ, Raina JB, Skarshewski A, Le XH, Butler MK, Stocker R, Seymour J, Tyson GW, Hugenholtz P (2016) Validation of picogram- and femtogram-input DNA libraries for microscale metagenomics. PeerJ 4: e2486 https://doi.org/10.7717/peerj.2486 
Sarantopoulou D, Tang SY, Ricciotti E et al. (2019) Comparative evaluation of RNA-Seq library preparation methods for strand-specificity and low input. Scientific Reports 9(1): 13477 https://doi.org/10.1038/s41598-019-49889-1

528

529

530

531

532

533

534

535

536

537

538

539

540

541

542

543

544

545

546

547

548

549

550

551

552

553

554

555

556

557

558

559

560

561

Sato MP, Ogura Y, Nakamura K, Nishida R, Gotoh Y, Hayashi M, Hisatsune J, Sugai M, Takehiko I, Hayashi T (2019) Comparison of the sequencing bias of currently available library preparation kits for Illumina sequencing of bacterial genomes and metagenomes. DNA Research 26(5): 391398, https://doi.org/10.1093/dnares/dsz017

Schmidl C, Rendeiro AF, Sheffield NC, Bock C (2015) ChIPmentation: Fast, robust, low-input ChIPseq for histones and transcription factors. Nature Methods 12(10): 963-965 https://doi.org/10.1038/nmeth.3542

Song Y, Milon B, Ott S et al. (2018) A comparative analysis of library prep approaches for sequencing low input translatome samples. BMC Genomics 19(1): 696. https://doi.org/10.1186/s12864-018-5066-2

Townsley BT, Covington MF, Ichihashi Y, Zumstein K, Sinha NR (2015) BrAD-seq: Breath Adapter Directional sequencing: A streamlined, ultra-simple and fast library preparation protocol for strand specific mRNA library construction. Frontiers in Plant Science 6: 366

van Rossum G, Drake FL (2009) Python 3 reference manual. Scotts Valley, CA: CreateSpace

Xavier C, Parson W (2017) Evaluation of the Illumina ForenSeq ${ }^{\mathrm{TM}}$ DNA Signature Prep Kit - MPS forensic application for the MiSeq $\mathrm{FGx}^{\mathrm{TM}}$ benchtop sequencer. Forensic Science International. Genetics 28: 188-194 https://doi.org/10.1016/i.fsigen.2017.02.018

Zhang J, Li J, Saucier JB, Feng Y, Jiang Y, Sinson J, McCombs AK, Schmitt ES, Peacock S, Chen S, Dai H, Ge X, Wang G, Shaw CA, Mei H, Breman A, Xia F, Yang Y, Purgason A, Pourpak A, Chen Z, Wang X, Wang Y, Kulkarni S, Choy KW, Wapner RJ, Van den Veyver IB, Beaudet A, Parmar S, Wong LJ, Eng CM (2019) Non-invasive prenatal sequencing for multiple Mendelian monogenic disorders using circulating cell-free fetal DNA. Nature Medicine 25(3): 439-447. doi: https://doi.org/10.1038/s41591-018-0334-x

Zheng XY, Gehring M (2019) Low-input chromatin profiling in Arabidopsis endosperm using CUT\&RUN. Plant Reproduction 32(1): 63-75 https://doi.org/10.1007/s00497-018-00358-1 
Tables and Figures

Table 1. Cost per sample for each technique

\begin{tabular}{|l|l|l|l|l|}
\hline Library & BrAD-Seq & TruSeq Nano & TruSeq ChIP & ThruPLEX \\
\hline Vendor & & Illumina & Illumina & Takara Bio \\
\hline Price (€) Euro & 2.36 & 44.31 & 78.75 & 52.07 \\
\hline Price (¥) JPY & 304 & 5,708 & 10,146 & 6,709 \\
\hline Price (\$) USD & 2.80 & 52.60 & 93.48 & 61.81 \\
\hline
\end{tabular}

565 Prices are shown for Japanese distributors with exchange rates at the time of submission to 566 indicate the relative costs alone. Exact costs depend on the distributors in each country.

567

568 Table 2. GC coverage bias across sliding windows of 10,000 bp in $A$. thaliana and $D$. 569 melanogaster

\begin{tabular}{|l|l|l|}
\hline Library & Slope $\left(\beta_{1}\right)$ in plants & Slope $\left(\beta_{1}\right)$ in flies \\
\hline 10 ng BrAD-Seq 3.6874 & 62.114 \\
\hline 10 ng TruSeq 9.2336 & 179.720 \\
\hline $10 \quad$ ng & 148.933 \\
\hline ThruPLEX 6.0395 & \\
\hline
\end{tabular}

$570 * \beta_{1}$ is the coefficient for linear regression for $\mathrm{y} \sim \beta_{1} \mathrm{x}+\beta_{0}$, where $\mathrm{y}$ is the coverage depth and $\mathrm{x}$ is

571 the GC content for each 10,000 bp sliding window of the genome. 
a)

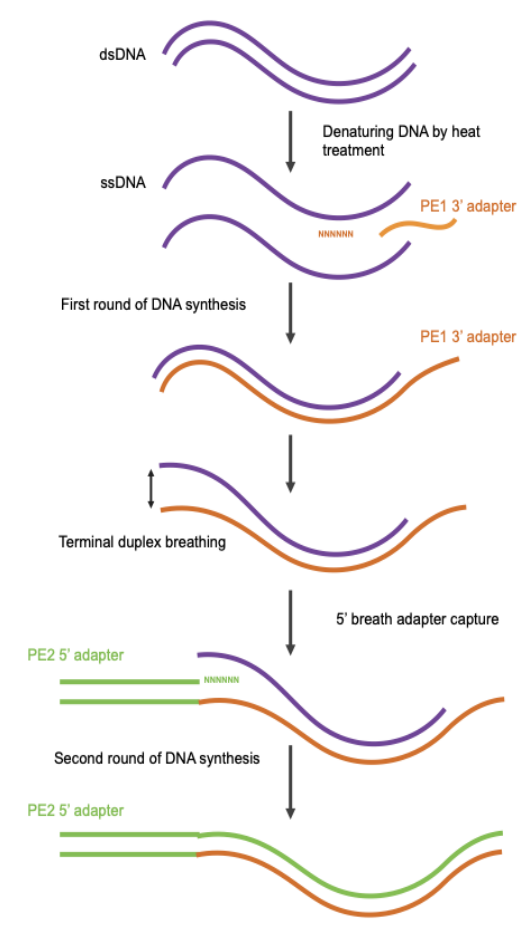

b)

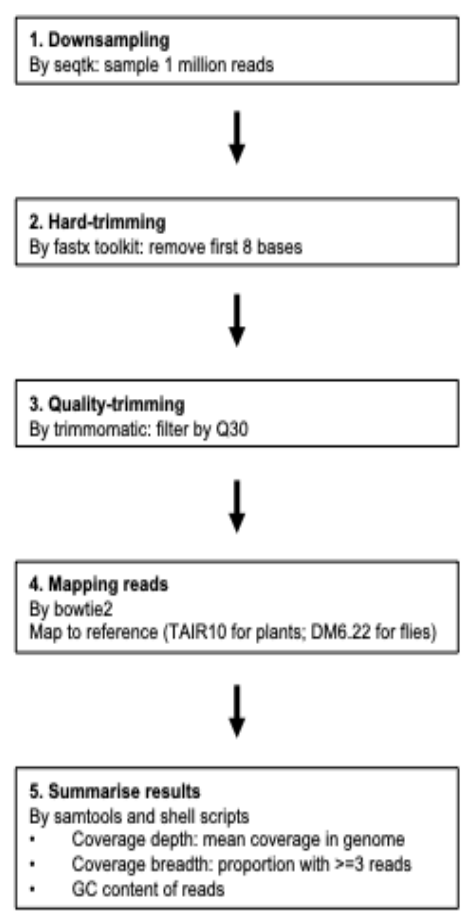

\section{Figure 1. Experimental and bioinformatics workflows for BrAD-Seq using genomic DNA}

a) Schematic representation of the breath capture technique for library preparation. Outline for how breath capture is performed to generate a library for next-generation sequencing from double-stranded DNA. b) Summary of steps in bioinformatics analysis. All samples were processed with the same parameters for comparison. 

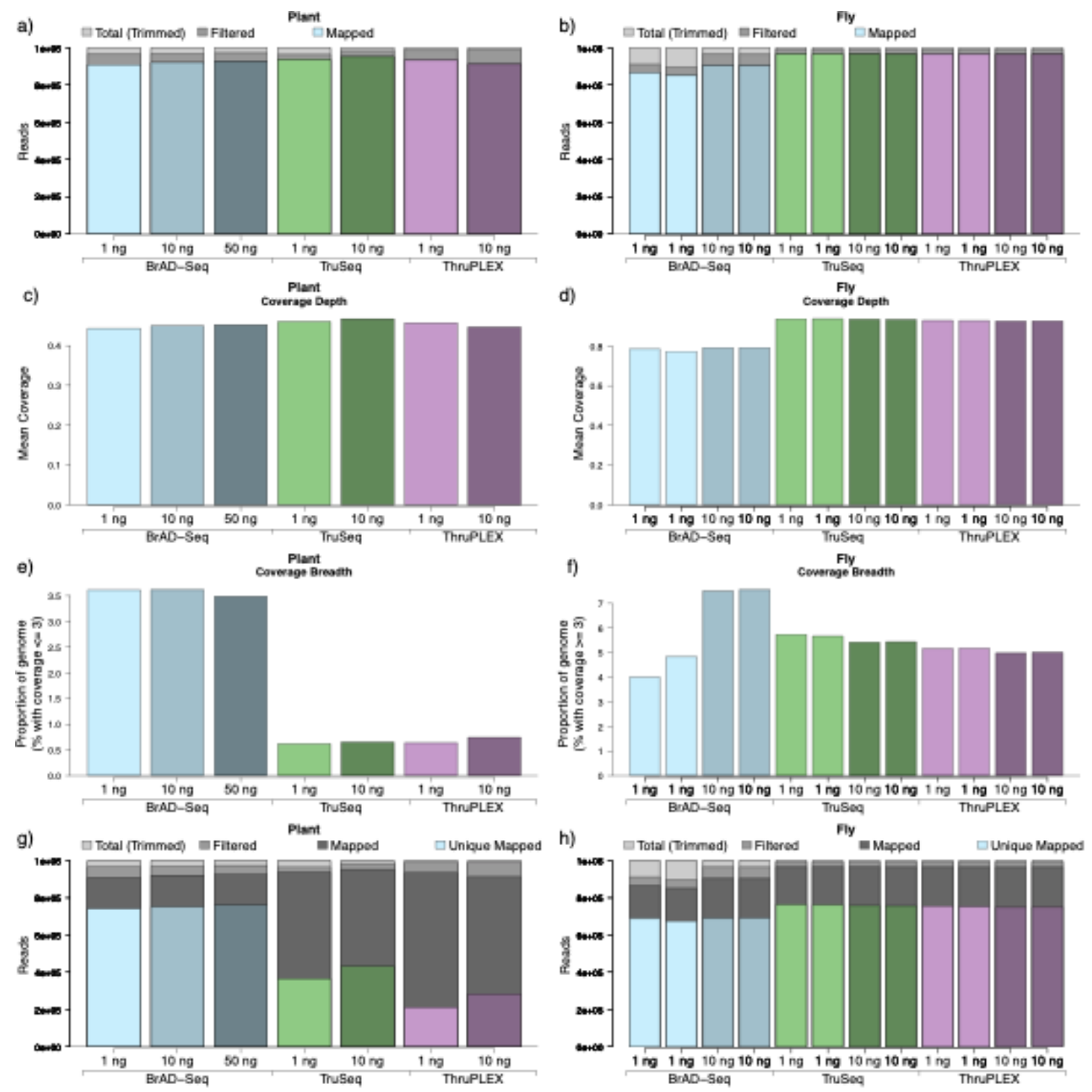

\section{Figure 2. Data processing summary for different library preparation techniques}

All libraries were downsampled to 1 million reads as single ends in read 1 . The number of reads remaining after filtering and mapping to the reference genome is shown for a) plant data, and b) fly data after downsampling. Mapped reads are shown as a proportion of total trimmed reads with unmapped quality-filtered reads in dark grey, as described in the key. The mean coverage depth for plant and fly data closely reflects the number of mapped reads. The coverage breadth (the proportion of the genome mapped by $\geq 3$ reads) for e) plant data, and f) fly data was often greater for many BrAD-Seq libraries. The unique mapping rate for g) plant data, and h) fly data shows that uniquely mapped reads were also higher in BrAD-Seq prepared using plant DNA and similar to other protocols using fly DNA. The fly samples were plotted in two replicates. 
594

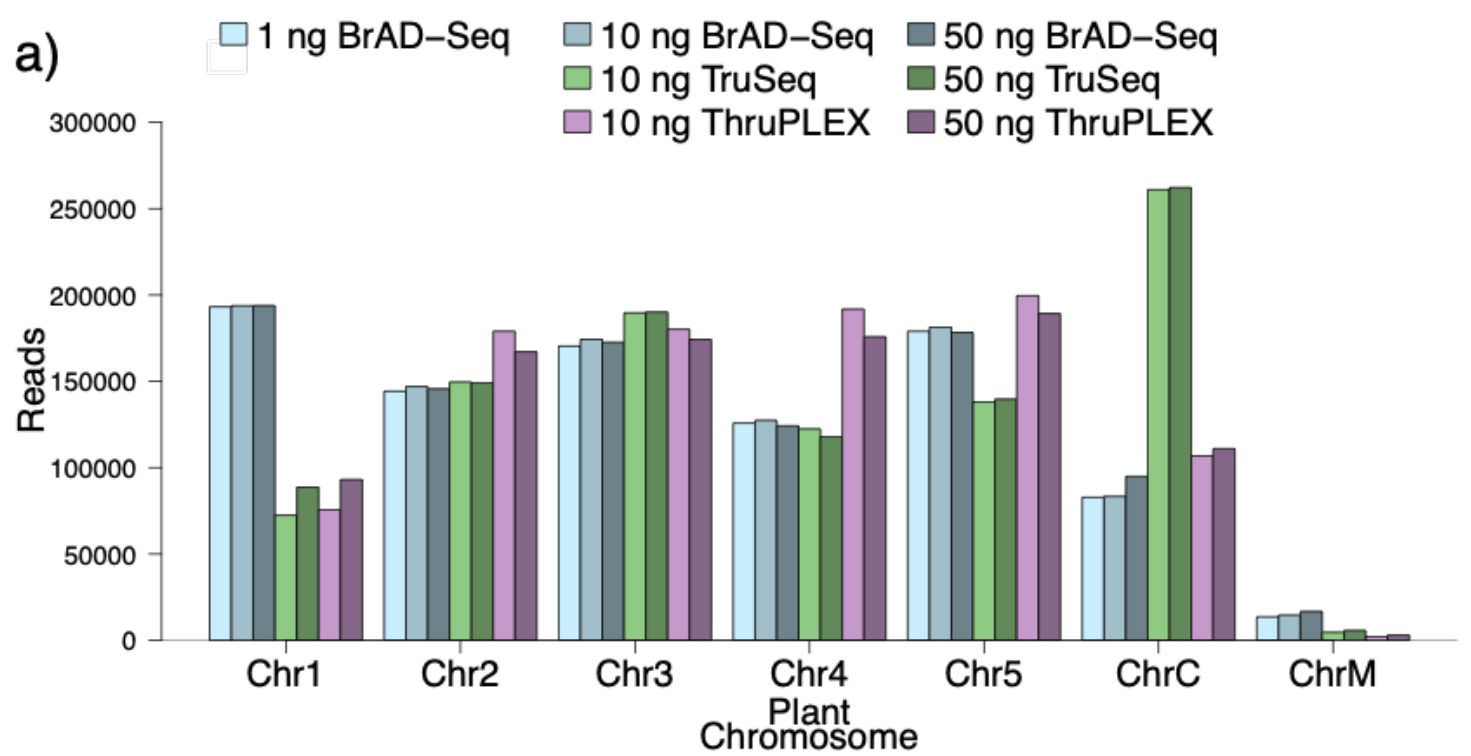

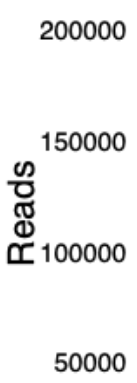

Figure 3. BrAD-Seq shows a relatively even distribution of reads per chromosome and for

598 All libraries were downsampled to 1 million reads, and the reads that mapped to each 599 chromosome were counted. The breakdown of reads per chromosome for a) plant data, and b) 600 fly data is shown. Unassembled scaffolds in the fly reference were excluded from the plot. In 601 plants, BrAD-Seq resulted in more reads for the longest chromosome (1), and TruSeq libraries 602 showed over-representation of chloroplast reads. Both TruSeqand ThruPLEX libraries contained 603 604 more mitochondrial reads (Fig. S2 for details). 
a)

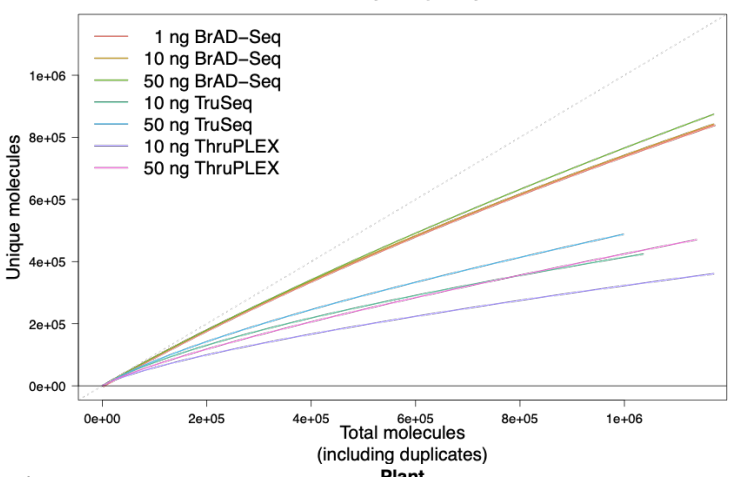

c)

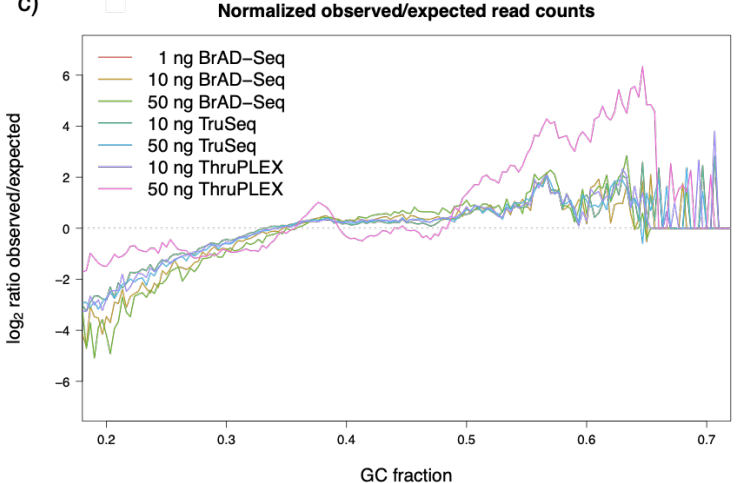

b)

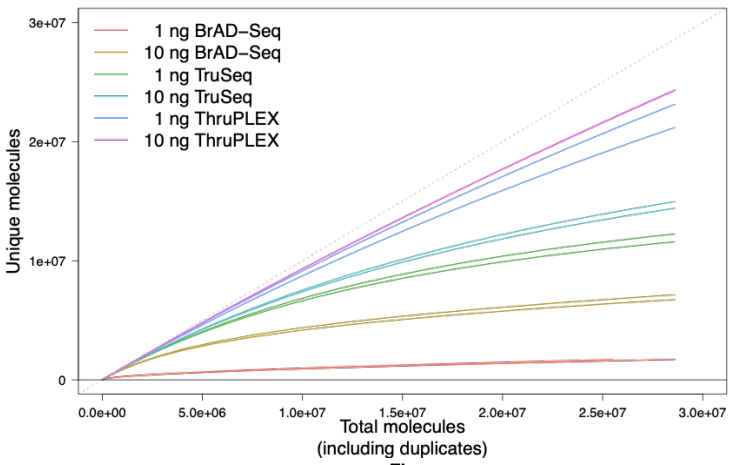

d)

Fly
Normalized observed/expected read counts

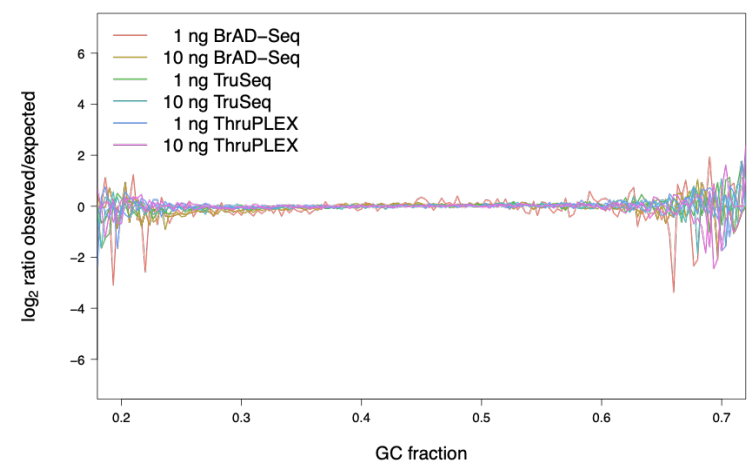

609 Library complexity is shown by plotting unique molecules by resampling the raw unmapped 610 reads as computed by preseq 3.0.2 for a) plant samples, and b) fly samples. Library complexity 611 was higher in BrAD-Seq for plant samples at all input amounts but was lower in fly samples in the 612 various replicates, especially for $1 \mathrm{ng}$ of BrAD-Seq libraries. GC bias, as computed by DeepTools

$613 \quad 3.5 .0$, is shown for $300 \mathrm{bp}$ fragments in c) plant, and d) fly samples. Illumina and Rubicon libraries 614 showed a greater bias towards high GC regions in the plant genome. A straight line at 0 indicates 615 no GC bias compared to the expected distribution of sampling of the reference genome. Some 616 variations at extreme values are expected because of sampling a smaller number of fragments. 
a)

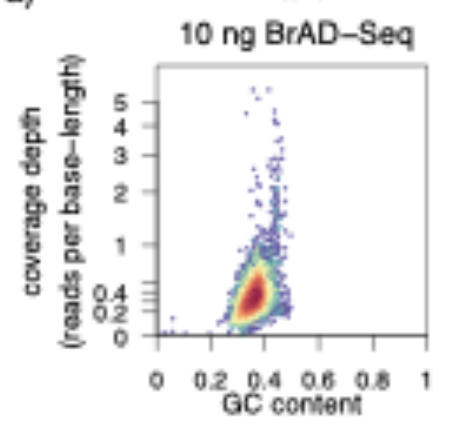

c) sliding window $(10,000 \mathrm{bp})$

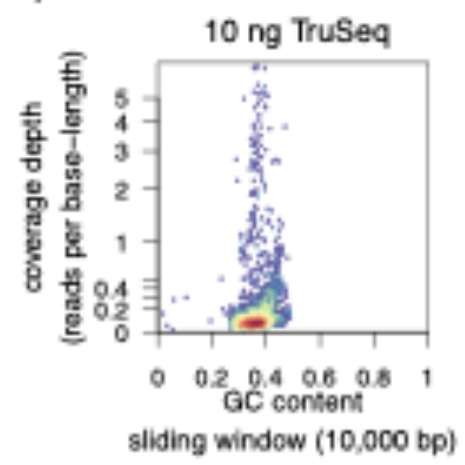

e)

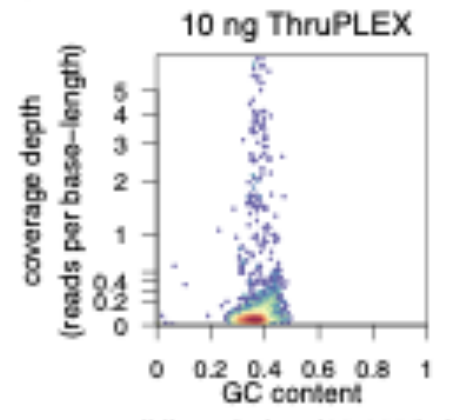

b)

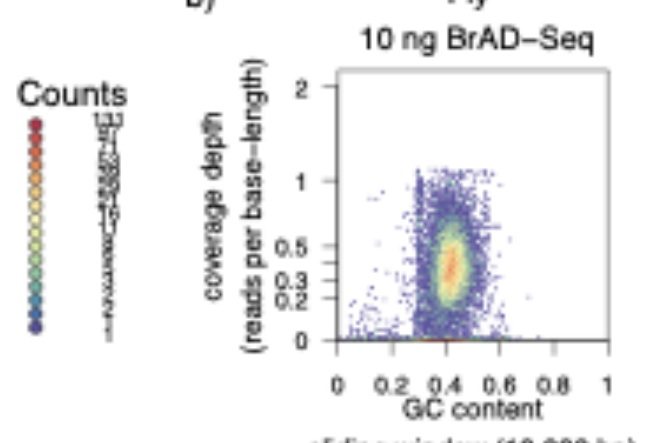

d)

siding window $(10,000 \mathrm{bp})$

Fly

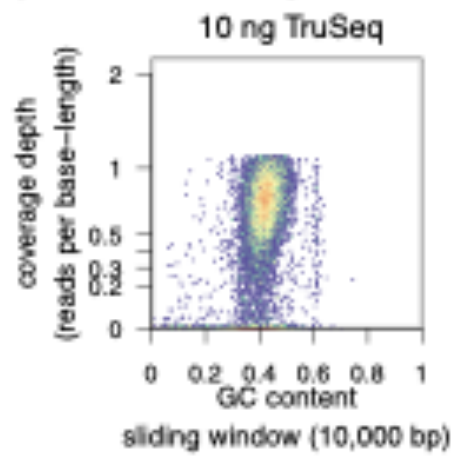

f)

Fly
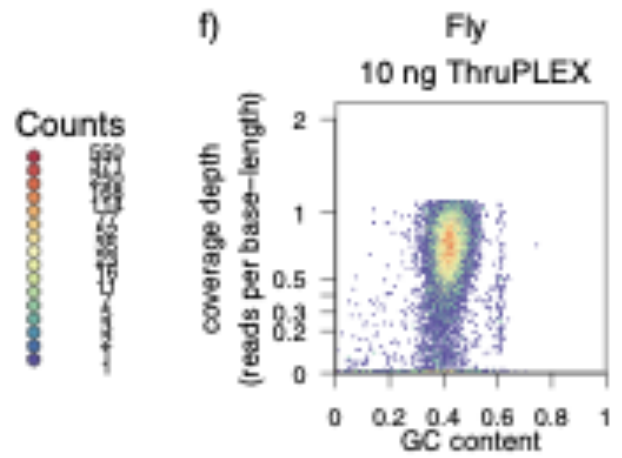

sliding window $(10,000 \mathrm{bp})$

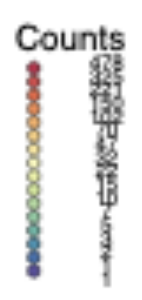

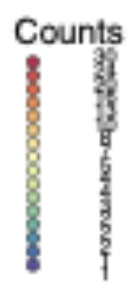
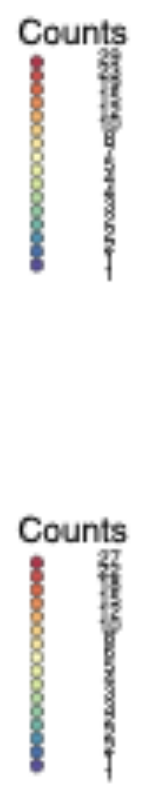

618

619

620

621

622

623

624

625

626

627

628

629

630

631

632 sliding window $(10,000 \mathrm{bp})$

Figure 5. Coverage depth in

\section{libraries}

Coverage depth varies with GC content at fine physical resolution, and the relationship differs between libraries. Reads were binned into 10,000 bp stepping windows in the genome and plotted on a log-scale against the GC base frequency for the same regions in the genome. Overlapping points were binned into hexagons and colored by density. Examples of a-b) BrADSeq, c-d) TruSeq, and e-f) ThruPLEX libraries are shown for 10 ng DNA input in plant (a, c, and e) and fly (b, d, and f) samples. TruSeq and ThruPLEX libraries had similar distributions and showed bias towards fragments with high GC content, particularly in plant samples. Summary statistics for GC content are presented in Tables S8 and S9. The linear regression results (Table 21) show that there was a greater linear effect of GC content on the read depth in the TruSeq or ThruPLEX libraries. In the low GC plant genome, Illumina and Rubicon libraries exhibited bias for GC-rich regions at a finer window resolution. 